\title{
Dermatology Research
}

\section{Analysis of Home Factors leading to the Development of Allergic Rhinitis in Children in Guangzhou, Southern China}

\author{
Zhong Jie*, Liu Li, Luo Renzhong, Huang Zhenyun and Sun Changzhi
}

Department of Otolaryngology, Guangzhou Women and Children's Medical Centre, China.

\section{*Correspondence:}

Zhong Jie, Department of Otolaryngology, Guangzhou Women and Children's Medical Center, Guangzhou Children's Hospital.318\# Ren Min Zhong Road, Yue Xiu District, Guangzhou, 510120, China, Tel: +86 13580334477; Fax: 02038076381 .

Received: 17 April 2021; Accepted: 04 May 2021

Citation: Jie Z, Li L, Renzhong L, et al. Analysis of Home Factors leading to the Development of Allergic Rhinitis in Children in Guangzhou, Southern China. Dermatol Res. 2021; 2(1): 1-7.

\section{ABSTRACT}

Objective: To analyse home factors leading to the development of Allergic Rhinitis (hereafter referred to as "AR") in children in Guangzhou, Southern China.

Methods: We selected children with a history of AR who attended the Otolaryngology Clinic of Guangzhou Women and Children's Medical Center from January 2020 to August 2020 to complete the "Home Environment Questionnaire for Children with Allergic Rhinitis" using an internet survey as well as telephone enquiries. We selected children who developed AR episodes during these 8 months as the case group and children who did not develop AR episodes as the control group to analyse independent influencing factors of home living environment leading to the development of AR in Children in Guangzhou.

Results: We completed a total of 667 questionnaires from parents of children with AR, and 644 questionnaires were validly returned, with a valid return rate of $96.55 \%$. There were 317 cases in the case group and 327 cases in the control group. Keeping pets, parents' smoking habits, bedroom orientation, living floor, frequency of plush toy cleaning, frequency of air conditioner cleaning, and frequency of bedding cleaning are all independent influencing factors of home environment leading to the development of AR in Children in Guangzhou (OR=3.618, 3.815, 7.576, $1.035,4.030,7.838,5.971, P<0.05)$.

Conclusions: A number of factors, inclusive of keeping pets, are all factors of home living environment leading to the development of AR in Children in Guangzhou, some of which require effective intervention, with particular attention to mites removal.

\section{Keywords}

AR, Home Environment, Allergens, Dust mites, Pets.

\section{Introduction}

Allergic Rhinitis (AR) is a chronic, non-infectious inflammatory disease of the nasal mucosa mediated by $\operatorname{IgE}$ that occurs in atopic individuals after exposure to allergens [1]. As one of common chronic pediatric diseases, AR often occurs in children and its episodes symptoms tend to recur. The incidence rate of $A R$ in children in China is approximately $10 \%$ and is increasing year by year [2]. With most children spending more than $80 \%$ of their time at home, AR for children is closely linked to the home environment $[3,4]$. Especially since the beginning of 2020 , with the outbreak of the COVID-19 epidemic and its worldwide spread, the time humans spent at home has increased significantly compared to the previous period, therefore, the influencing factors of home living 
environment are worthy of further analysis. Guangzhou is located in the southeastern coastal region of China, with high temperatures and humidity all year round. There is a gap in research on related factors of home living environment leading to the development of $\mathrm{AR}$ in children in this region. In this research, we selected some parents of children who met the requirements as research objects, and collected data by means of a multidimensional questionnaire to conduct a preliminary survey and analysis of influencing factors of home environment leading to the development of AR in Children in Guangzhou, in the hope of providing some data support for the progress of this work. It is reported as follows:

\section{Objects and Methods}

Research Objects

We selected survey data from parents of children with a history of AR who attended the Otolaryngology Clinic of Guangzhou Women and Children's Medical Centre (Zhujiang New Town Campus, Children's Campus and Maternal and child hospital area) from January 2020 to August 2020 to conduct analysis. This research was reviewed and permitted by the Medical Ethics Committee of Guangzhou Women and Children's Medical Centre.

\section{Criteria for inclusion}

(i) Children aged 2 to 16 years with a history of AR diagnosis;

(ii) Parents of children with at least a primary school education, able to communicate properly and complete the questionnaire used in this research by telephone or online;

(iii) Children who had lived in the Guangzhou for more than 24 consecutive months.

\section{Criteria for exclusion}

(i) Parents with visual and hearing impairments or mental abnormalities that make communication difficult;

(ii) Children participating in other medical research at the same time;

(iii) Parents refusing to take part in this survey;

(iv) Parents or children with other situations who need to be excluded as researchers considered.

\section{Research Methods}

\section{Questionnaire Design}

The researchers designed the "Home Environment Questionnaire for Children with Allergic Rhinitis" based on their work experience. The questionnaire consists of two main sections: general information about Children with AR and information about home environment. A pre-test in hospital showed good reliability, Cronbach's $\alpha=0.863$.

\section{Questionnaire Completion}

Taking the opportunity of children with AR attending the clinic, "Home Environment Questionnaire for Children with Allergic Rhinitis" was distributed in the form of a QR code scan, which was answered by parents of children through the WeChat network, based on the informed consent of the parents of children. Children with a history of AR diagnosis but who had not developed the disease were selected in the hospital's electronic medical records management system, and their parents were contacted by telephone and asked to complete "Home Environment Questionnaire for Children with Allergic Rhinitis" by telephone enquiries.

\section{Data Entry}

After quality control of the questionnaires completed by the different routes, the researchers used a two-persons, two-machines method to enter the data obtained into the Epidata 3.02 software and checked them to ensure the accuracy of the data obtained.

\section{Grouping Methods}

Children with AR episodes from January 2020 to August 2020 were selected as the case group and children without AR episodes were selected as the control group.The diagnostic criteria for AR episodes were referred to "the Guidelines for the diagnosis and treatment of Allergic Rhinitis "[]]: (i) Symptoms: at least two of nasal congestion, clear runny nose, sneezing and nasal itching with sudden and recurrent episodes lasting more than one hour per day; (ii) Physical signs: pale, edematous nasal mucosa, heavy watery discharge, enlarged turbinate, allergic dark circles under the eyes and allergic folds in severe cases; (iii) Positive serum atopic immunoglobulin E or skin prick test result; and (iv) At least one allergen result of $(++)$ or more.

\section{Observed Indicators}

Description of the Return of the Questionnaires.

The data from the case and control groups were compared to analyse the independent influencing factors of home environment leading to the development of AR in Children in Guangzhou through single-factor as well as multi-factors methods in turn.

To analyse the predictive value of the risk of episodes in children with AR by a multi-factors integrated model.

\section{Statistical Processing}

SPSS 24.0 software is used for statistical processing. Data of normally distributed measures are expressed as $(x \pm s)$, and the results of counting data are expressed using the number of cases (n) and percentage (\%). Data of normally distributed measures are compared using independent sample t-test, and $\chi^{2}$ test is used for comparison of counting data. Logistic regression is used for multi-factors analysis, and the predictive value is counted using the method of Receiver Operating Characteristic (ROC) curve, and the difference is considered statistically significant at $P<0.05$.

\section{Results}

Return of the Questionnaires

A total of 667 questionnaires were completed for parents of children with AR, of which 23 questionnaires were considered invalid because they were incomplete or perfunctory, so 644 questionnaires were validly returned, with a valid return rate of $96.55 \%$.

Analysis of Single-Factor of Home Environment Leading to the Development of AR in Children

There was a total of 317 cases in the case group and 327 cases in 
the control group. The results of the single-factor analysis show that keeping pets, parents' smoking habits, bedroom orientation, living floor, frequency of plush toy cleaning, frequency of air conditioner cleaning, and frequency of bedding cleaning may be related factors of home environment leading to the development of AR in children in Guangzhou (Table 1).

Analysis of Multi-Factors of Home Environment Leading to the Development of AR in Children

Using whether the child had an AR episode as the dependent variable, of which $0=$ control group and $1=$ case group, a multi- factors analysis was performed on the items that showed positive results in the single-factor analysis above, and the results of the categorical variable assignments are presented in Table 2. All variables are included using the "entry" method, and the results show that keeping pets, parents' smoking habits, bedroom orientation, living floor, frequency of plush toy cleaning, frequency of air conditioner cleaning, and frequency of bedding cleaning are all independent influencing factors of home environment leading to the development of AR in Children in Guangzhou (OR=3.618, $3.815,7.576,1.035,4.030,7.838,5.971, P<0.05)$ (Table 3).

Table 1: Analysis of Single-Factor of Home Environment Leading to the Development of AR in Children.

\section{Related factors}

Age $(\mathrm{x} \pm \mathrm{s})$ (years)

Gender $[\mathrm{n}(\%)]$

Male

Female

Body weight $(\mathrm{x} \pm \mathrm{s})(\mathrm{kg})$

Disease course of AR $(x \pm s)$ (years)

Father's age $(\mathrm{x} \pm \mathrm{s})$ (years)

Mother's age ( $\mathrm{x} \pm \mathrm{s})$ (years)

Number of persons in the family ( $\mathrm{x} \pm \mathrm{s})$ (persons)

Living area $(\mathrm{x} \pm \mathrm{s})\left(\mathrm{m}^{2} /\right.$ person $)$

Living floor $(\mathrm{x} \pm \mathrm{s})$ (Floor)

Living region $[\mathrm{n}(\%)]$

Urban

Rural

Monthly family income $(\mathrm{x} \pm \mathrm{s})(\mathrm{RMB} /$ person)

Years of parental education $(\mathrm{x} \pm \mathrm{s})$ (years)

Parents having smoking habits $[\mathrm{n}(\%)]$

Yes

No

Keeping pets [n(\%)]

Yes

No

The child's bedroom orientation [ $\mathrm{n}(\%)]$

South

Non-south

Number of plush toys owned by the child ( $\mathrm{x} \pm \mathrm{s}$ ) (toys)

Frequency of plush toys cleaning [n(\%)]

$\geq$ once/week

$<$ once/week

Number of air conditioners in the home $(\mathrm{x} \pm \mathrm{s})$ (air conditioners)

Frequency of air conditioners cleaning [n(\%)]

$\geq$ once /month

$<$ once/ month

Frequency of bedding cleaning [n(\%)]

$\geq$ once/week

$<$ once/week

Table 2: Assignments of Categorical Variables.

\begin{tabular}{|c|}
\hline Categorical Variables \\
\hline Keeping pets \\
\hline Parents having smoking habits \\
\hline Bedroom orientation \\
\hline Frequency of plush toy cleaning \\
\hline Frequency of air conditioner cleaning \\
\hline Frequency of bedding cleaning \\
\hline
\end{tabular}

Dermatol Res, 2021 ents having smoking habits

quency of air conditioner cleaning

Frequency of bedding cleaning

\section{Case group $(\mathrm{n}=317)$ Control group $(\mathrm{n}=327)$}

$$
5.79 \pm 2.42
$$

173 (54.57)

144 (45.43)

$19.65 \pm 8.22$

$2.01 \pm 0.78$

$33.68 \pm 5.93$

$30.27 \pm 4.97$

$2.93 \pm 1.42$

$21.60 \pm 5.36$

$5.38 \pm 2.02$

209 (65.93)

108 (34.07)

$3799.78 \pm 756.93$

$13.53 \pm 4.04$

$112(35.33)$

205 (64.67)

89 (28.08)

228 (71.92)

117 (36.91)

$200(63.09)$

$2.11 \pm 0.56$

189 (59.62)

$128(40.38)$

$1.86 \pm 0.61$

99 (31.23)

218 (68.77)

231 (72.87)

$86(27.13)$
$5.98 \pm 2.31$

159 (48.62)

$168(51.38)$

$20.13 \pm 9.53$

$1.98 \pm 0.83$

$33.47 \pm 6.08$

$29.87 \pm 5.11$

$3.02 \pm 1.32$

$20.97 \pm 4.77$

$8.32 \pm 2.51$

$211(64.53)$

$116(35.47)$

$3861.59 \pm 803.55$

$13.66 \pm 3.93$

86 (26.30)

241 (73.70)

$43(13.15)$

284 (86.85)

91 (27.83)

$236(72.17)$

$2.05 \pm 0.47$

227 (69.42)

$100(30.58)$

$1.79 \pm 0.55$

71 (21.71)

256 (78.29)

$270(82.57)$

57 (17.43)

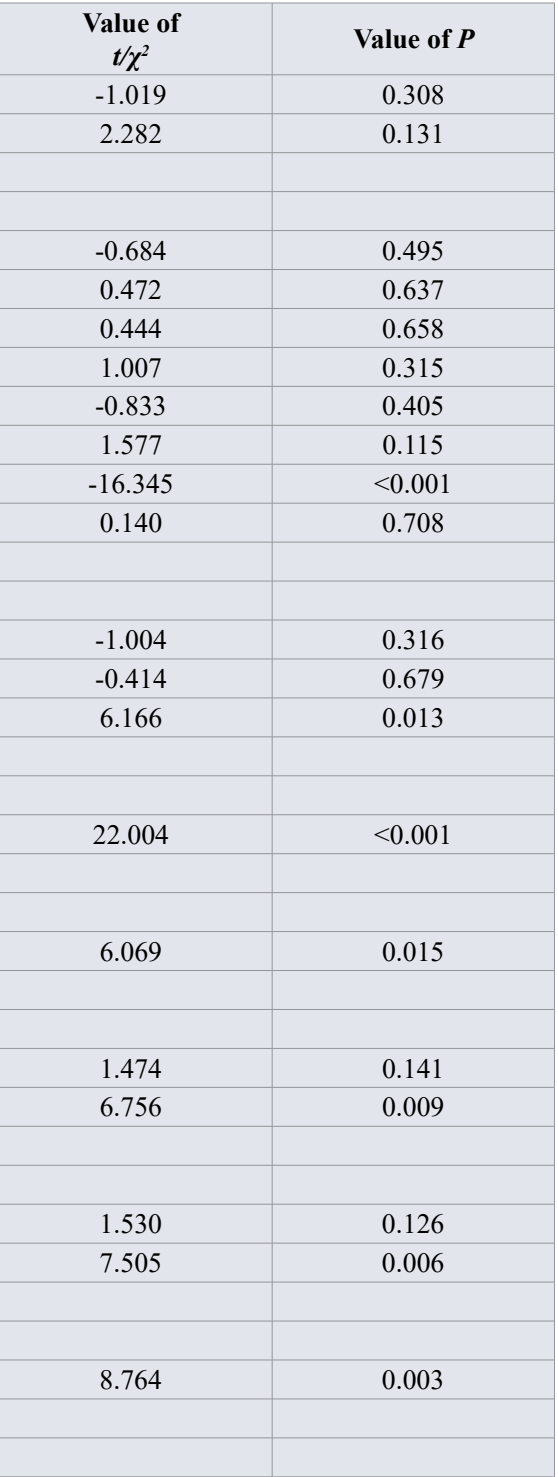

\section{Assignments}

$0=$ Yes, $1=$ No

$0=$ Yes, $1=$ No

0 = South, $1=$ Non-south

$0=\geq$ once/week, $1=<$ once/week

$0=\geq$ once $/$ month, $1=<$ once/ month

$0=\geq$ once/week, $1=<$ once/week 
Table 3: Analysis of Multi-Factors of Home Environment Leading to the Development of AR in Children.

\begin{tabular}{|c|c|c|c|c|c|}
\hline Related factors & $\begin{array}{c}\text { Regression coefficient } \\
\beta\end{array}$ & Standard error & $\begin{array}{c}\text { Wald } \\
\chi^{2}\end{array}$ & Value of $P$ & Value of OR $(95 \% \mathrm{CI})$ \\
\hline Keeping pets & 1.286 & 0.474 & 7.351 & 0.006 & $3.618(1.428-9.167)$ \\
\hline Parents having smoking habits & 1.339 & 0.468 & 8.192 & 0.004 & $3.815(1.525-9.544)$ \\
\hline Bedroom orientation & 2.025 & 0.603 & 11.286 & $<0.001$ & $7.576(2.324-24.691)$ \\
\hline Living floor & 0.035 & 0.000 & 6542.021 & $<0.001$ & $1.035(1.034-1.036)$ \\
\hline Frequency of plush toy cleaning & 1.394 & 0.270 & 26.598 & $<0.001$ & $4.030(2.373-6.846)$ \\
\hline Frequency of air conditioner cleaning & 2.059 & 0.983 & 4.389 & 0.036 & $7.838(1.141-53.804)$ \\
\hline Frequency of bedding cleaning & 1.787 & 0.867 & 4.245 & 0.039 & $5.971(1.090-32.689)$ \\
\hline
\end{tabular}

Table 4: The Predictive Value of the Risk of Episodes in Children with AR by a Multi-Factors Integrated Model.

\begin{tabular}{|l|c|c|c|c|c|}
\hline Items & Best cut-off value (\%) & Area under ROC curve & Predictive sensitivity (\%) & Predictive atopy (\%) & Jorden Index (\%) \\
\hline Value of P in Logistic model & 66.69 & 0.773 & 57.10 & 84.10 & 41.20 \\
\hline
\end{tabular}

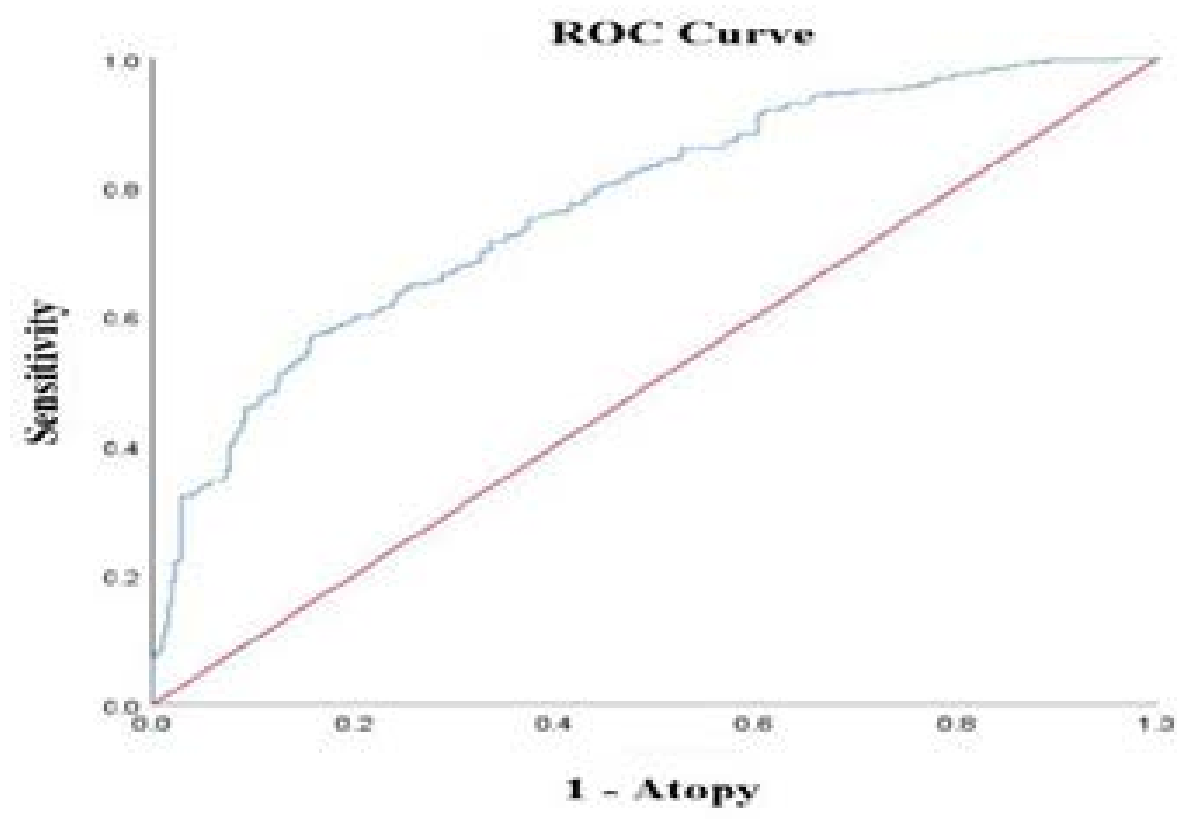

Chart 1: Predictive ROC Curve for the Risk of Episodes in Children with AR by Value of P in Logistic Model.

The Predictive Value of the Risk of Episodes in Children with AR by a Multi-Factors Model

Using the Logistic model obtained in Table 3 to predict the risk of episodes in children with AR. The best cut-off value and the Jorden index are $66.69 \%$ and $41.20 \%$ respectively (Table 4 and Chart I).

\section{Discussions}

With the development of human society, the population incidence rate of AR in children is increasing year by year and has become one of the most prevalent diseases endangering the respiratory system health of children [6]. Zhao Jing et al. found that the incidence rate of AR in children was $14.5 \%, 20.4 \%$ and $7.8 \%$ in 2011 in a survey of children allergic diseases in the central areas of three major cities in China, Beijing, Chongqing and Guangzhou, respectively [7]. While in the 2017 AR survey research of children in Chongqing, Hu Sijie et al. found that the self-reported incidence rate of AR among children in Chongqing was $28.5 \%$, which was higher than the finding reported by Zhao Jing et al. six years ago
(20.4\%) [8]. AR not only affects the quality of life of the children, but also increases the economic burden on the individual, family and society [9-10].

The interaction between the body's genetic susceptibility and environmental factors is the underlying cause of the development of AR [11]. Genetic susceptibility does not change at one's will, but we can reduce AR episodes by controlling and intervening in environmental factors. Most children spend more than $80 \%$ of their time at home and rhinitis in children is closely linked to the indoor environment [3-4]. Especially since the beginning of 2020, the outbreak of the COVID-19 epidemic has significantly increased the amount of time humans spent at home compared to the previous period, therefore, this research focuses on the home living environment of children with AR.

A self-designed questionnaire is used in this research due to the lack of established questionnaires as well as data constants at present. The questionnaire was found to have good reliability in 
the pre-test and the results were considered reliable. Considering the need to survey cases as well as parents of children in both case and control groups, we used two different questionnaire methods in this research. An on-site survey was used for children who attended the Otolaryngology clinic as results of AR episodes, and a telephone survey strategy was used for parents of children with AR without episodes. Although this survey method did not guarantee uniformity of survey way to a certain extent, it was chosen because of the nature of the disease under research and in order to find good control children for matching. With the researcher's explanation and assistance, the vast majority of parents completed the questionnaires well.

The results of this research show that age, gender, number of family members, living area, living region (urban or rural), family income level and parents' education level are not statistically different between the case and control groups $(P>0.05)$ and do not correlate significantly with factors of home environment leading to the development of AR in children in Guangzhou. In a multicenter research including seven large cities, Jiao Cai et al. pointed out that the incidence rate of AR in children living in urban areas of China was significantly higher than that in rural or suburban areas of China [12]. The findings on the living urban factors in this research differ from those mentioned above, which may be related to the fact that this research was only conducted on children in Guangzhou and was not representative of findings in multiple other regions or cities. At the same time the findings on urban factors in Guangzhou may indeed be different from those in Chongqing, Shanghai, Beijing and other regions. This will require further findings from future research's with larger sample sizes.

The results of this research also show that keeping pets, parents' smoking habits, bedroom orientation, living floor, frequency of plush toy cleaning, frequency of air conditioner cleaning, and frequency of bedding cleaning are independent influencing factors of home environment leading to the development of AR in Children in Guangzhou $(p<0.05)$. Exposure to susceptible allergens is an important factor in the development of AR [11], with the most common indoor allergens being dust mites, cockroaches, animal hair and fungi, etc. [13]. Each of these seven home independent factors listed in the results of this research is significantly correlated with each of these four major categories of common indoor allergens.

In this research, keeping pets was found to be one of the home environment factors leading to the development of AR in children. Cat and dog hair dander are common allergens in modern life where more and more families prefer to keep pets [14]. Playfulness with pets, combined with the presence of natural shedding of animal hair dander, significantly increases the chances of exposure to animal hair dander allergens in children with AR. Even though keeping pets has some psychological benefits for adults and children and contributes to the healthy physical and mental development of children [15], it is still recommended that families of children with AR who have been tested positive for cat or dog hair allergens avoid keeping such pets. For families who do not want to give up keeping pets, scholars have two recommendations: (i) To prohibit pets from entering the bedroom to avoid animal hair dander staying in the bedroom; (ii) To use a dust collector with a HEPA system and insist on collecting dust from the sofa, curtains, bedding, carpets and other indoor areas where animal hair dander tends to accumulate at a frequency of more than once per week [14].

The results of this research show that parents' smoking habits are a risk factor for the development of AR in children. Even though our government is increasing its efforts to ban smoking, the dangers of second-hand smoke still exist in some homes. Passive smoking has become one of causes for many respiratory system diseases such as asthma in children at home [16]. Children's respiratory system defenses are inadequate and harmful substances from tobacco smoke in environment can trigger AR episodes by promoting allergen-atopic IgE production, inducing Th2 cytokine differentiation and stimulating the release of histamine from nasal mucosal cells [17]. Therefore, for the health of children, parents are strongly advised not to smoke and to avoid exposing their children to second-hand smoke.

The results of this research show that bedroom orientation and living floor are both factors of home environment leading to the development of AR in Children in Guangzhou. Bedroom orientation and living floor are related to the humidity of the living environment. There is a strong association between the humidity in the home and the development of AR. Researches from Jiao Cai et al. showed that exposure to signs of dampness in the home is a risk factor for AR and asthma in children, with $1.46 \%$ of children with $\mathrm{AR}$ in the past year having an association with visible mold and mildew in their current residence [12]. The climate in the south is relatively damp and southern families self-report a higher proportion of their exposure to indoor dampness than northern families [18]. Guangzhou is located in a subtropical region with a relatively damp climate all year round. If the children's bedrooms are oriented to the south, daylighting and ventilation would be better. If living at a higher floor, the humidity of the indoor air would drop relatively as the height of the building rises. Meanwhile, reducing dampness in the living environment also reduces the risk of AR episodes in children by reducing the breeding of dust mites. Based on the results of this research, it is recommended that families of children with AR who are able to do so should try to choose a living building with south-facing bedrooms and a floor above the 8th floor, which may be more beneficial to the health of children with AR.

Most of the children with AR who participated in this research owned at least one plush toy, with a higher percentage of girls in particular owning a plush toy. Plush toys vary in quality, and if they are not cleaned properly, they can easily breed mold and dust mites, etc. [19]. The results of this research show that the frequency of plush toy cleaning more than once per week may help reduce the incidence rate of AR in children. For families of children with AR, plush toys should be avoided as much as possible and replaced with plastic toys or other toys of non-allergenic materials. If children do rely on plush toys, they should be reduced in number 
and cleaned more frequently, and should be kept off the bedroom bed as much as possible.

Air conditioner is an important factor contributing to the development of asthma as well as AR [20]. The results of this research show that the frequency of air conditioner cleaning is one of the home factors leading to the development of AR in Children. On the one hand, air conditioners regulate the indoor temperature and humidity and even have the function of air filtration and purification, thus reducing the risk of indoor allergen exposure [21]. On the other hand, air conditioner filters are a high gathering place for dust mites [19]. Many families tend to neglect the cleaning of air conditioner filters, often not cleaning them or only cleaning them once a year, which is far from enough. In order to reduce mites gathering in air conditioner filters, it is recommended to clean them once per week during the season when an air conditioner in use [20]. At the same time, the climate in Guangzhou is hot and damp, and air conditioners are used for a longer time during the year, while the indoor cold air when the air conditioner is in use tends to stimulate the blood vessels in nasal mucosa, leading to the episodes of AR symptoms. In our 2014 research, our team found that the number of children with AR who attended the clinic in Guangzhou increased in summer compared to other seasons, which was related to the increased use of air conditioners in summer [22]. Therefore, it is recommended that families of children with AR reduce the use frequency and use hours of air conditioners, and that the difference between the indoor and outdoor temperatures when air conditioners are in use should not be too great, keeping at around $27^{\circ} \mathrm{C}$ indoor. It is also recommended to keep the habit of cleaning air conditioner filters once per week.

The results of this research show that the frequency of bedding cleaning was a home factor leading to the development of AR in Children, and that enhanced bedding cleaning was effective in reducing dust mite's concentration. There is a dose-dependent relationship between environmental dust mite's exposure and AR symptoms, and reducing the concentration of dust mites in the environment can help reduce the development of AR in children [23]. The best living environment for dust mites is an environment with a temperature of $20^{\circ}$ to $25^{\circ} \mathrm{C}$ and a relative humidity of more than $55 \%$. The climate in the southern region is more suitable for dust mites to reproduce and breed. Dust mites are the main allergen in children with AR in Guangzhou, with up to $90 \%$ of children with AR having a positive skin prick test for dust mites [22]. One research found that dust mites concentrations were much higher in bedding than in the living room, and that air conditioner filters, bedding, plush toys, soft furnishings and other places are the most common places for dust mites [19,24], so it is important to focus on mite's removal in the bedroom. Water or hot air above $50^{\circ}$ can kill dust mites directly and most mite's removal measures can significantly reduce the concentration of dust mites in the environment, but a single mites removal measure is often limited in its effectiveness and a combination of various mite's removal measures is required [25]. Combining the results of several previous researches $[21,24,25]$, the authors summarize the following five comprehensive measures of mites removal: (i) To use anti-mite knitted fabrics or use anti-mite covers to wrap mattresses, pillows and bedding, use non-allergenic bedding (highcount cotton, silk quilts, latex pillows); (ii) To clean bed sheets and covers weekly with water above $55^{\circ}$, use sunlight exposure (firstly cover secondly pat and thirdly collect: To use a black plastic bag to cover them in sunlight exposure for two hours or more, remove the black plastic bag and pat them, and collect dust in one direction for two minutes with a dust collector with HEPA system), and pay attention to clean the mattress; (iii) To avoid dampness in the bedroom (30\% to 50\% humidity) and keep the air flowing; (iv) To minimize the accumulation of household clutter, not to use carpets and shutters, avoid plush toys, and should choose wood floor, tile or polished stone floor; and (v) To clean the air conditioner filters regularly (once per week when in use).

There are also certain pities in this research: although the data were selected from three hospital districts, the overall sample size is still relatively limited and scientific validation and data analysis of the questionnaire design used in this research on a larger scale of population are yet required to be conducted, all of which are yet to be explored in depth when suitable opportunities are selected in the future.

In summary, a number of factors, inclusive of keeping pets, are all factors of home living environment leading to the development of AR in Children in Guangzhou, some of which require effective intervention, with particular attention to mite's removal.

\section{Reference}

1. Rhinology Group in Editorial Board of Chinese Journal of Otolaryngology - Head and Neck Surgery, Rhinology Group in Otolaryngology - Head and Neck Surgery Branch of Chinese Medical Association. Guidelines for the Diagnosis and Treatment of Allergic Rhinitis (Tianjin, 2015). Chinese Journal of Otolaryngology - Head and Neck Surgery. 2016; 51: 6-24.

2. Kong W J, Chen J J, Zheng Z Y, et a1. Prevalence of allergic rhinitis in 3-6-year-old children in Wuhan of China. Clin Exp Allergy. 2009; 39: 869-874.

3. Fan G, Xie J, Yoshino H, et al. Environmental conditions in homes with healthy and unhealthy school children in Beijing, China. Build Environ. 2017; 112: 270-84.

4. Reynolds L A, Finlay B B. Early life factors that affect allergy development. Nat Rev Immunol. 2017; 17: 518-528.

5. Rhinology Group in Editorial Board of Chinese Journal of Otolaryngology - Head and Neck Surgery, Rhinology Group and Pediatrics Group in Otolaryngology - Head and Neck Surgery Branch of Chinese Medical Association, Editorial Board of the Chinese Journal of Pediatrics. Guidelines for the Diagnosis and Treatment of Allergic Rhinitis in Children (Chongqing, 2010). Chinese Journal of Otolaryngology Head and Neck Surgery. 2011; 46: 7-8.

6. Li C W, Chen $\mathrm{D} \mathrm{H}$, Zhong $\mathrm{J} \mathrm{T}$, et al. Epidemiological characterization and risk factors of allergic rhinitis in the 
general population in Guangzhou city in China. PLoS One. 2014; 9: e114950.

7. Zhao Jing, Bo Juan, Shen Kun Ling, et al. A Questionnaire Survey on Allergic Diseases in Children Aged 0-14 Years in the Central Urban Areas of Beijing, Chongqing and Guangzhou. Chinese Journal of Pediatrics. 2011; 49: 740-744.

8. Hu Sijie, Yao Hongbing, Peng Yanling, et al. Epidemiological Survey and Analysis of Allergic Rhinitis in Children in Chongqing. Chongqing Medicine. 2017; 46: 4700-4704.

9. Thanaviratananich S, Cho S H, Ghoshal A G, et al. Burden of respiratory disease in Thailand:results from the AP-BORD observational study. Medicine (Baltimore). 2016; 95: e4090.

10. Yoo K H, Ahn H R, Park J K, et al. Burden of respiratory disease in Korea:an observational study on allergic rhinitis,asthma, COPD, and rhinosinusitis. Allergy Asthma Immunol Res. 2016; 8: 527-534.

11. Wang De Yun. Risk factors of allergie rhinitis genetic or environmental. Therapeutics and Clinical Risk Management (Print). 2008; 1: 15-23.

12. Jiao Cai, Baizhan L i, Wei Y u, et al. Household dampnessrelated exposures in relation to childhood asthma and rhinitis in China: A multicentre observational study. Environment International. 2019; 126: 735-746.

13. Cheng L, Chen J, Fu Q, et al. Chinese society of allergy guidelines for diagnosis and treatment of allergic rhinitis. Allergy Asthma Immunol Res. 2018; 10: 300-353.

14. Yuan Feng. Logistic Regression Analysis of Risk Factors Associated with the Development and Prognosis of Allergic Rhinitis. Chinese Journal of Otolaryngology - Skull Base Surgery. 2017; 23: 266-270.

15. Luan M. Chu, Donna C. Rennie, Donald W. Cockcroft, et al. Prevalence and determinants of atopy and allergic diseases among school-age children in rural Saskatchewan, Canada. Annals of Allergy, Asthma Immunology. 2014; 113: 430-439.

16. Li Y, Jiang Y, Li S, et al. Pre and postnatal risk factors in relation to allergic rhinitis in school-aged children in China. PLoS One. 2015; 10: e0114022.

17. Diaz Sanchez D, Rumold R, Gong H Jr. Challenge with environmental tobacco smoke exacerbates allergic airway disease in human beings. J Allergy Clin Immunol. 2006; 118: 441-446.

18. Rui Xiaoqing, Jiang Yanrui, Zhu Qi, et al. Risk Factors and Quality of Life Analysis of Allergic Rhinitis Combined with Asthma in Preschool Children. Chinese Journal of Otolaryngology-Head and Neck Surgery. 2020; 27: 263-266.

19. Raulf M, Bergmann K C, Kull S, et al. Mites and other indoor allergens: from exposure to sensitization and treatment. Allergo J Int. 2015; 24: 68-80.

20. Wilson J M, TAE P. Home Environmental Interventions for House Dust Mite. J Allergy Clin Immunol Pract. 2018; 6: 1-7.

21. Manuyakorn W, Padungpak S, Luecha O, et al. Assessing the efficacy of a novel temperature and humidity control machine to minimize house dust mite allergen exposure and clinical symptoms in allergic rhinitis children sensitized to dust mites: a pilot study. Asian Pac J Allergy Immunol. 2015; 33: 129135.

22. Zhong Jie, Liu Dabo, Huang Zhenyun. Analysis of the Results of Inhaled Allergen Skin Prick Test in 2136 Children with Allergic Rhinitis in Guangzhou. Chinese Journal of Otolaryngology-Head and Neck Surgery. 2014; 21: 481-485.

23. Wang Y, Xiong L, Yin X, et al. House dust mite allergen levels in households and correlation with allergic rhinitis symptoms. Am J Rhinol Allergy. 2014; 28: 193-196.

24. Causer S, Shorter C, Sercombe J. Effect of floorcovering construction on content and vertical distribution of house dust mite allergen, Der p I. J Occup Environ Hyg. 2006; 3: 161168.

25. Nurmatov U, van Schayck C P, Hurwitz B, et al. House dust mite avoidance measures for perennial allergic rhinitis: an updated cochrane systematic review. Allergy. 2012; 67: 158165. 